\title{
Ring resonator based SOI biosensors
}

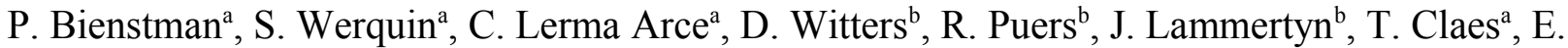 \\ Hallynck $^{\mathrm{a}}$, J.-W. Hoste ${ }^{\mathrm{a}}$, D. Martens ${ }^{\mathrm{a}}$ \\ ${ }^{a}$ Ghent University, Photonics Research Group, Dept. of Information Technology, \\ Sint-Pietersnieuwstraat 41, 9000 Gent, Belgium \\ ${ }^{\mathrm{b}}$ KU Leuven, MeBioS Biosensor group, Department of Biosystems, Willem De Croylaan 42, 3001 \\ Leuven, Belgium
}

\begin{abstract}
In this paper, two recent advances in silicon ring resonator biosensors are presented. First, we address the problem that due to the high index contrast, small deviations from perfect symmetry lift the degeneracy of the normal resonator mode. This severely deteriorates the quality of the output signal. To address this, we discuss an integrated interferometric approach to give access to the unsplit, high-quality normal modes of the microring resonator. Second, we demonstrate how digital microfluidics can be used for effective fluid delivery to nanophotonic microring resonator sensors fully constructed in SOI.
\end{abstract}

Keywords: Silicon photonics, biosensors, ring resonators, peak splitting, digital microfluidics

\section{INTRODUCTION}

The high index contrast of the silicon-on-insulator platform allows for fabrication of micrometer size sensors ${ }^{1}$. However, it also limits the quality of the resonances by introducing an intrinsic mode-splitting. Small deviations from perfect symmetry lift the degeneracy of the normal resonator modes. This severely deteriorates the quality of the output signal. The quality of the resonances is of utmost importance to determine the performance of the microrings as a biosensor. We suggest an integrated interferometric approach to give access to the unsplit, high-quality normal modes of the microring resonator.

Second, we demonstrate how digital microfluidics can be used for effective fluid delivery to nanophotonic microring resonator sensors fully constructed in SOI.

\section{INTERFEROMETRIC PEAK SPLITTING COMPENSATION}

The high index contrast of the SOI-platform causes high confinement of the optical fields in the waveguides, which makes the microrings very sensitive to changes on the waveguide surface. This explains their very high sensitivity, but at the same time, waveguide roughness causes scattering of the guided light. This degrades the quality factor of the resonances and can ultimately lead to splitting of a resonance ${ }^{2}$. Because the detection limit of a microring biosensor is directly related to the quality of the ring resonance, a high Q-factor is of primordial importance in sensing applications. In this paper, we present an integrated interferometric approach to resolve the resonance splitting of a microring resonantor on a single chip, resulting in a significant improvement of the resonance quality.

\section{Origin of resonance splitting}

A perfectly symmetric microring resonator mode is twofold degenerate. Both clockwise $(\mathrm{CW})$ and counterclockwise (CCW) propagation are possible in the microring and both modes are uncoupled. This degeneracy is lifted when the $\mathrm{CW}$-mode and CCW-mode become coupled. Surface roughness on the waveguide edges and the proximity of bus waveguides for microring interrogation form deviations from circular symmetry. These deviations cause forward propagating light to scatter back into the opposite direction, exciting a CCW-mode from a CW-mode and vice versa. Standing-wave modes as a symmetric and antisymmetric superposition of the traveling waves can be considered as the 
new eigenmodes of the system. They will however, no longer be degenerate as a consequence of the symmetry breaking coupling. If the linewidth of the resonance is small enough to distinguish both modes, the resonance splitting will be visible in the output signal. This occurs for high quality resonances. Evidence of this effect is provided in figure 1, which shows both the pass-port and add-port spectrum of a microring in add-drop configuration. Only the input port is excited, so ideally, no power is present in the CCWmode and the add-port remains dark. The measurement shows that backscattering in the microring waveguide cannot be neglected, resulting in significant power in the add-port and resonance-splitting in the pass-signal.

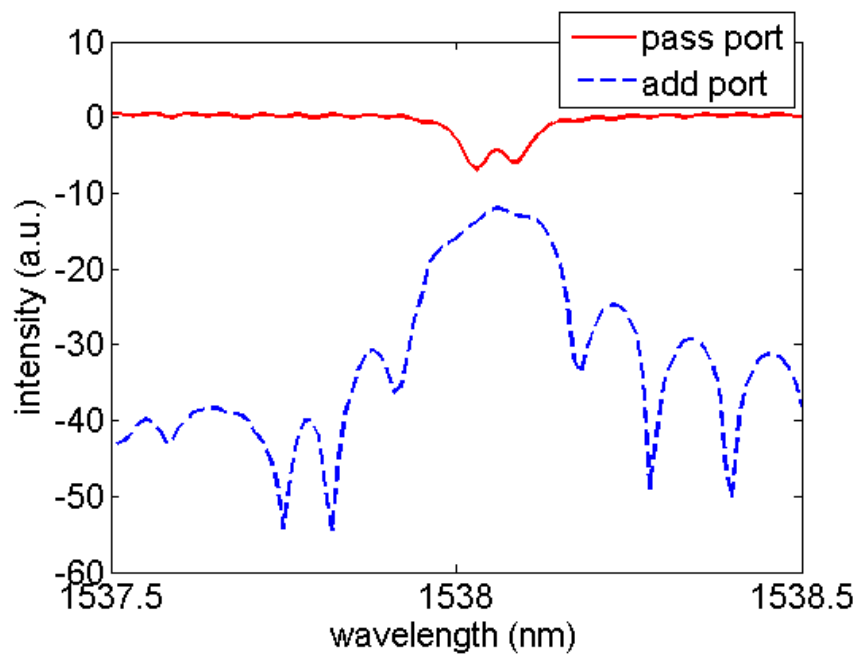

Figure 1: Measured spectra showing resonance splitting and backscattered power

\section{Integrated interferometric circuit}

As demonstrated in [3], an interferometric approach can be used to access the normal modes of the microring resonator in an output signal. We have implemented this in an integrated circuit on a single SOI-chip. A layout of the circuit is provided in figure 2. Vertical grating couplers are used to couple light from a tunable laser light source into the circuit and collect the power at the output. The input light excites a CW-mode in the microring resonator, a CCW-mode is excited as a consequence of mode-coupling. The normal modes of the microring are a symmetric and antisymmetric superposition of the $\mathrm{CW}$ and $\mathrm{CCW}$-mode. Both fields are combined in a multimode interferometer (MMI), If we design the circuit such that the phase difference between the reflected and transmitted wave equals a multiple of 0 or $\pi$, we see that the signals in output one and two are proportional to the normal modes of the resonator. At the same time, output three is proportional to the pass-signal of the microring resonator in the all-pass configuration. This means we have access to the unsplit, high-Q normal modes of the cavity. If the detection limit of a biosensor is limited by the quality-factor of the resonance, this provides a tool to improve the detection limit significantly. Higher resonator Q-factors give rise to lower detection limits. 


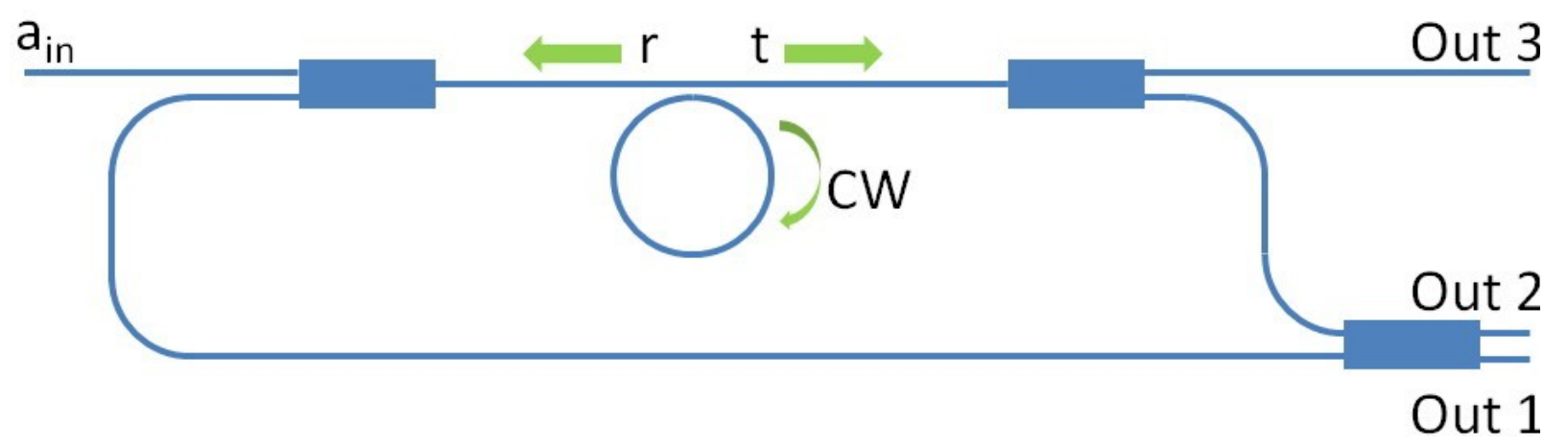

Figure 2: layout of the integrated interferometric circuit

\section{Experimental results}

The circuit from figure 2 is designed and processed in a CMOS pilot line at imec. Using the vertical in- and outputcouplers on the waveguides, the chip can easily be measured in a fiber-to-fiber configuration. For the junction regions where waveguides are combined and splitted, MMI 2x1 and MMI 2x2 couplers are used. The coupling from the microring to the waveguide is ensured by weak evanescent coupling to a neighbouring bus waveguide. To obtain the measured spectra, a SANTEC TSL-510 Tunable laser source is used to generate the input signals. Output intensities are measured by a HP-8153 optical power meter. The laser wavelength is swept in 10pm steps during recording of the power.

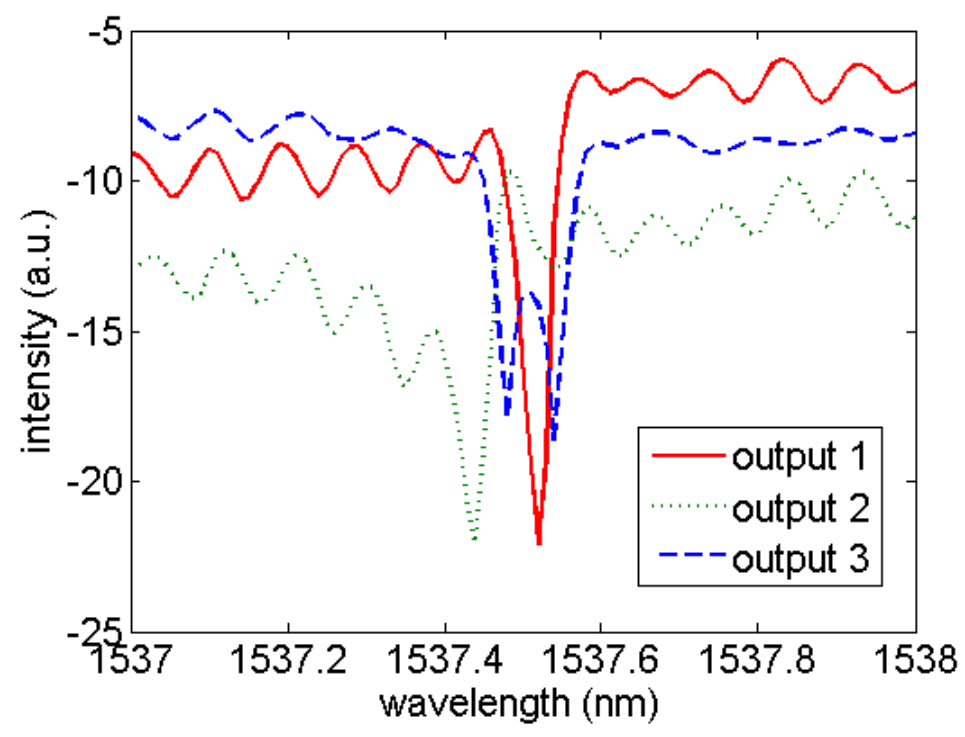

Figure 3: Measured output spectra of the circuit. Output one clearly shows the improvement of the resonance shape compared to the all-pass signal in output three.

Figure 3 shows the recorded spectra at the three outputs of the circuit. Output one and two are proportional to the normal modes of the resonator, output three returns the all-pass spectrum. We clearly see the all-pass spectrum shows severe splitting of $60 \mathrm{pm}$ in the resonance. This values is comparable to $3 \mathrm{~dB}$-bandwidth of the normal mode in output one. This result shows an almost twofold improvement of the resonance quality from output three to output one. Notice the distinct asymmetrical shape of the resonance in output two which makes unambiguous definition of a Q-value difficult. Additionally, the extracted normal modes do not correspond exactly with the normal modes we can distinguish in the all-pass signal. This non-ideal behaviour is a consequence of fabrication variations. The $2 \times 2 \mathrm{MMI}$ is especially sensitive 
to this and is not exactly balanced as a result. Also the phase relation between the reflected and the transmitted wave differs slightly from the required value of $\mathrm{k} \pi$. This can be solved by careful tuning of the waveguide structures.

\section{INTEGRATION WITH DIGITAL MICROFLUIDICS}

A crucial component in most of the photonic biosensors is the transducer that can transform a refractive index change in its environment to a measurable change in its optical transmission. Microring resonator sensors stand out as prime candidates for such transducers for achieving high performance in a robust manufacturable manner.

However, not only the transducer but also the microfluidic system is an essential element for such sensing systems. The use of microfluidic channels for delivery of the sample in continuous flow to the sensor surface is one of the most critical steps in the integration of biosensors and microfluidics for point-of-care applications. This integration is not straightforward, since the interface with the outside world needs special attention, and care needs to be taken to avoid leaks and channel clogging.

Digital microfluidics is an emerging technology similar to the more established technology of microfluidic channels but unique because all elementary fluidic operations (droplet dispensing, splitting, merging, transport, and mixing) are performed on reconfigurable paths of buried actuation electrodes with high flexibility without the need of moving parts such as pumps or valves.

Here, we demonstrate how digital microfluidics can be used for effective fluid delivery to nanophotonic microring resonator sensors fully constructed in SOI.

\section{Digital microfluidic system}

The cross-section of the EWOD lab-on-a-chip is shown in Figure 4.
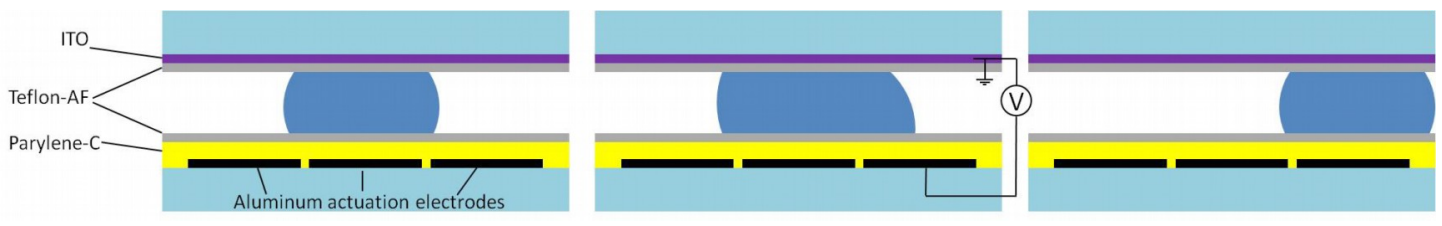

Figure 4. A diagram of the cross-section of an EWOD lab-on-a-chip and the electrowetting principle. When a voltage is applied to an actuation electrode, the droplet is attracted toward this activated region due to the generated imbalance in interfacial tension near the surface above the actuated electrode.

The digital microfluidic platform was produced by means of standard photolithographic techniques. For the bottom part of the chip, a 100-nm thick aluminum layer was sputtered on a glass wafer and subsequently patterned using standard photolithographic techniques. All electrodes were $1.4 \times 1.4 \mathrm{~mm}^{2}$. The aluminum layer was covered by a $2.8 \mu \mathrm{m}$ dielectric Parylene-C layer deposited using chemical vapor deposition. This insulating layer was rendered hydrophobic by spin-coating a layer of Teflon-AF (300 nm thickness) on top. The top part of the digital lab-on-a-chip consisted of a glass plate coated with a $120 \mathrm{~nm}$ layer of transparent indium tin oxide (ITO) which was made hydrophobic with a Teflon-AF layer of approximately $300 \mathrm{~nm}$. Standard tape with a thickness of $80 \mu \mathrm{m}$ was used as spacer between top and bottom plates.

Droplets were sandwiched between the two plates. To activate the electrowetting-on-dielectric mechanism, a 130V DC actuation voltage was used, while the electrode actuation sequence, activation time and relaxation time were controlled by means of homemade programs in MATLAB and LabView. When a voltage is applied to the system, a surface tension gradient at the droplet surface is evoked which attracts the droplet towards the activated region.

\section{Nanophotonic ring resonator sensors, chip layout and setup.}

The photonic chip was fabricated in silicon-on-insulator with $2 \mu \mathrm{m}$ buried oxide and a 220 -nm silicon top layer with CMOS-compatible 193-nm optical lithography and dry etching. The resonators consist of 450-nm-wide single-mode waveguides, with $5 \mu \mathrm{m}$ bend radius, $2 \mu \mathrm{m}$ long directional couplers and a gap of $180 \mathrm{~nm}$ between the waveguides. The layout of the chip is illustrated in Figure 5. 


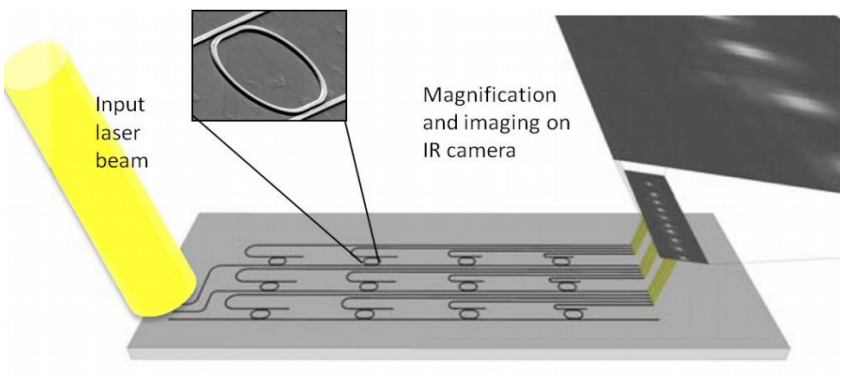

Figure 5: Chip layout top view. Four rings are connected to one common input waveguide, each of them having a dedicated drop signal port. Three of these four ring series are placed independently next to the other. A SANTEC TSL-510 tunable laser was used as a light source. The three input waveguides are simultaneously addressed through vertical grating couplers with a 2 mm-wide collimated beam from a tunable laser source. The output signals of the ring resonators are near-vertically coupled to free space by means of integrated grating couplers and are imaged with an infrared camera.

\section{Integrating both technologies}

The SOI chip containing the array of ring resonator sensors described above was incorporated into the digital microfluidic system by replacing the top glass plate of the platform. Figure 6 illustrates how the chip was placed up-side down, squeezing the liquid droplets against the bottom plate containing the buried electrodes.

In order to guarantee the hydrophobicity of the SOI chip surface, which is crucial for performing droplet manipulations efficiently, and at the same time ensure the contact of the sensors with the droplets, a layer of Teflon-AF ( $300 \mathrm{~nm}$ thickness) was coated on the SOI chip and subsequently patterned. Hereto, the Teflon-AF surface was activated with $\mathrm{O}_{2}$-plasma to allow photoresist to be spincoated on top. This thin layer of photoresist was then patterned by using standard photolithography to expose the regions covering the ring resonators. Next, the Teflon-AF covering the ring resonators was locally removed with reactive ion etching using $\mathrm{O}_{2}$-plasma. Some ring resonators were left covered with Teflon-AF in order to provide a reference to compensate for environmental drift.

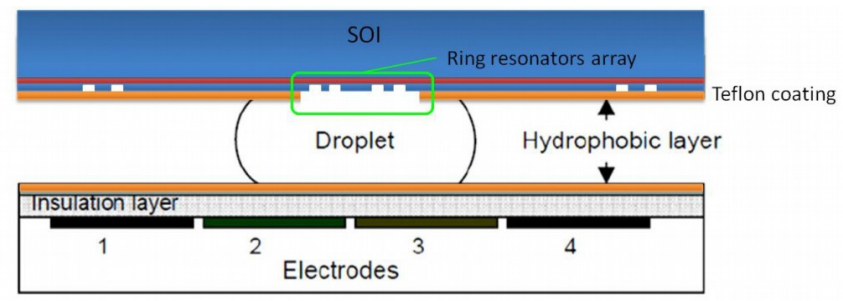

Figure 6: Incorporation of the SOI chip containing an array of microring resonator sensors into the digital microfluidic system by replacing the top glass plate of the platform ${ }^{4}$.

Grating couplers were used to couple the light from a tunable laser into the chip and couple it out to be detected by an infrared camera. A new aspect with respect to our previous work ${ }^{1}$ is that now, since the chip is placed up-side down, light needs to be coupled in and out through the $750-\mu \mathrm{m}$ thick silicon substrate. Silicon is considered practically transparent for the wavelength used $(1.55 \mu \mathrm{m})$.

However, to reduce the scattering of the rough substrate surface and to facilitate the alignment of the laser beam and the detection of the light coupled out from the chip, a few simple processing steps were done in advance: the silicon substrate was thinned down to $300 \mu \mathrm{m}$ by chemical mechanical grinding and afterwards a chemical mechanical polishing step was performed in order to attain a smooth surface. 


\section{Bulk sensing experiments}

As a proof-of-principle to show the capabilities of the combined system, we measured the sensitivity for refractive index changes of aqueous solutions and compared it with previous measurements for the same array of sensors using typical microfluidics based on microchannels and the simulations for bulk refractive index changes done in Fimmwave. Three sets of experiments were performed with sodium chloride, glucose and ethanol. For each of these compounds, 3 different droplets with different concentrations were prepared.

The measurements were performed as follows, e.g. for the sodium chloride solutions: a droplet of DI-water was transported on the digital microfluidic platform to the sensors area. Our system started measuring. Subsequently, the droplet of water was moved, leaving a free route for the second droplet with a first sodium chloride concentration. Since the read-out system performs continuous measurements in time, we measured in air during the switching of droplets, causing a big shift in the resonance wavelength, which was discarded. When the second droplet reached the sensing area, the measured signal will shift again to a similar wavelength as measured during the previous droplet, since the refractive index of different watery solutions does not differ to a large extent.

This same process was repeated for measuring glucose and ethanol concentrations in DI-water in order to confirm the behavior of the system for different refractive index materials and samples, while guaranteeing reproducibility.

Analyzing these shifts as a function of the refractive index unit (RIU) of the droplets, we observed a very close correspondence between the simulations and the measurement results (Figure 7). $78 \mathrm{~nm} / \mathrm{RIU}$ is the sensitivity that these ring resonators show for refractive index change simulations, which was also measured and proved using the typical microfluidcs system based on microchannels. The measurements performed in this digital microfluidic system show a sensitivity of $77 \pm 0.6 \mathrm{~nm} / \mathrm{RIU}$, which shows that the performance of the transducer does not suffer from being integrated in a digital microfluidics system.
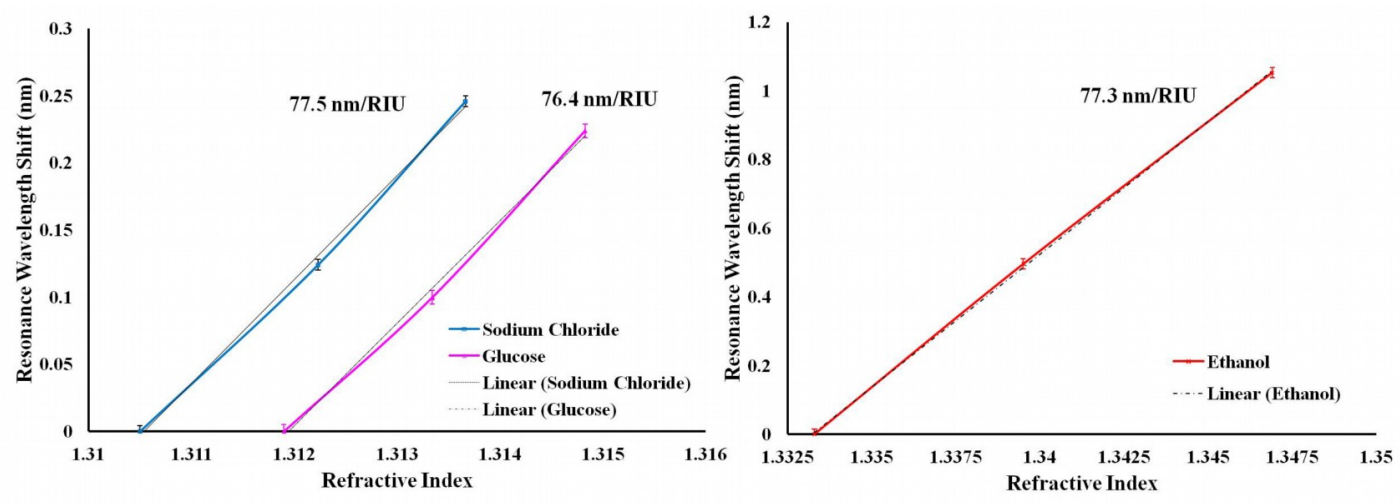

Figure 7. Three experiments with different sodium chloride, glucose and ethanol concentrations were performed. Each line corresponds to one of these experiments. Error bars indicate the standard deviations based on 3 repetitions. The sensitivity is determined to be $77 \pm 0.6 \mathrm{~nm} / \mathrm{RIU}$.

\section{REFERENCES}

K. De Vos, J. Girones Molera, T Claes, Y. De Koninck, S. Popelka, E. Schacht., R. Baets, P. Bienstman, IEEE Phot Journal, 1(4): 225-235, 2009.

T. J. Kippenberg, S. M. Spillane, and K. J. Vahala, "Modal coupling in traveling-wave resonators", Optics letters, vol. 27, no. 19, pp. 1669-71, Oct. 2002.

J. Knittel, T. G. McRae, K. H. Lee, and W. P. Bowen, "Interferometric detection of mode splitting for whispering gallery mode biosensors", Applied Physics Letters, vol. 97, no. 12, p. 123704, 2010.

N. Vergauwe, D. Witters, F. Ceyssens, S. Vermeir, B. Verbruggen, B, Puers, J. Lammertyn, J. Micromech. Microeng. 21: 054026, 2011. 